tures common to both processes; and this, in turn, suggests that the known characteristics of one process may be used to predict the properties of the other; for example, solid explosives possess instabilities which, when triggered, produce the explosion. The fact that viscous flow is governed by the same basic heat flow equation then implies that regions of viscous flow in the Earth (for example, the asthenosphere) should also possess inherent instabilities which, if triggered, should produce thermal runaways.

Insofar as thermal runaways in the Earth were proposed by Gruntfest more than a decade ago, this prediction by analogy may not seem particularly useful. Fortunately, however, there is more to be gained, for, as Anderson and Perkins point out, thermal explosions have been studied in some detail, and the knowledge obtained thereby may be used to extend the analogy with viscous flow. Pursuing this line of attack and making reasonable assumptions about the properties of the heat flow equation, Anderson and Perkins conclude that the potential for thermal runaways in the asthenosphere does indeed exist and that the time taken for the temperature to rise to the very high values necessary would probably be a few tens of million years after the initiating event.

Once such a thermal event has been triggered, the hotter material will rise towards the lithosphere in what Anderson and Perkins choose to call a surge. Some of the stronger surges would actually reach the bottom of the lithosphere but would probably not penetrate it. Instead, the surging material would spread out horizontally and then sink as it cooled, although completely circular paths would not be produced thereby because any given surge would die out in about the same time as it took to grow. On the other hand, the time scale involved here is tens of million of years, so that regions of high temperature (hot spots) would be expected to persist near the lithosphere-asthenosphere boundary for periods significant even in geological terms.

The geological implications of such a situation are clearly important. For example, although the hot asthenospheric material itself may not penetrate the lithosphere, its presence immediately beneath would facilitate partial melting in the upper mantle and lower crust, and thus crustal igneous activity. The significant point to note here is that zones of partial melting produced in this way would be distributed irregularly and would thus not necessarily bear any regular temporal or spatial relationship to steadystate subduction processes. Acceptance of surges would therefore mean that it would be no longer necessary to force complex arrangements of igneous activity into patterns dictated by plate tectonic models. The case that Anderson and Perkins have in mind here is the wide extent and complex patterns of Cainozoic igneous activity in the south-western United States. The problems involved in attempting to explain this activity in plate tectonic terms have already been emphasised by several workers; Noble et al. (Bull. geol. Soc. Amer., 84, 1393; 1973), for example, noted that the late Tertiary volcanic field of south central Nevada contains materials generated from different sources or different levels or both.

Finally, if thermal runaways produce surges in the asthenosphere, what triggers them? Thermal explosions in solids are triggered by thermal impulses and, by analogy, asthenospheric 'explosions' could be initiated by friction at the lithosphere-asthenosphere boundary, mantle plumes or changes in activation energy (for example, by dilution with a suitable volatile). Anderson and Perkins, however, also draw attention to other possible triggers which are less directly thermal and more concerned with changing asthenospheric flow patterns. Examples are the breakup of continents and the replacement of subduction zones by transform faults. It is clear, for instance, that the encroachment of the Pacific ridge on Atlantic plate must have profoundly affected flow patterns in the region and thus severely disrupted the asthenospheric thermal gradients.

\section{Models of the Earth}

\section{from $P$. G. Richards}

THE classical subjects of theoretical geophysics dominated a symposium held from June 25 to July 5 at Cambridge: no neat and easily solved novelties here, but the staples of mantle convection, dynamo motions in the core, scattering theory, weather prediction and earthquake source mechanisms.

At a session honouring Sir Edward Bullard, who is retiring from the Cambridge chair of Geodesy and Geophysics, F. Gilbert and A. Dzeiwonski (University of California, San Diego and Harvard) described notable new levels of sophistication in their study of the Earth's free oscillations. Several hundred new modes have been identified, and the inversion of 1066 data yields an improved Earth model in which the source mechanisms of two deep focus earthquakes can be retrieved accurately. Their results back up a controversial claim, made earlier, that the source region experiences compression, beginning about $80 \mathrm{~s}$ before the origin time as determined from body wave observations.

Since 1972, when the International Union of Geodesy and Geophysics sponsored its first symposium on mathematical geophysics, there has clearly been progress in the understanding of the Earth's core. M. Chinnery (Lincoln Laboratory, MIT) gave a new formulation for finding static deformations of an Earth model with a compressible fluid core, showing that current debate and major disagreements in the literature on this subject can be resolved by working with bulk properties in the fluid (density, pressure) rather than with particle displacements. D. Crossley (Memorial University, Newfoundland) presented results for the gravitational undertones in a subadiabatic core of a rotating Earth, finding that eigenperiods are confined to the range $0-12 \mathrm{~h}$. The written versions of these two papers are awaited with interest, for the basic problem has challenged Love, Jeffreys, Pekeris, and many another of the sharpest theoreticians in geophysics.

In weather prediction and seismic wave propagation, it is now possible to recognise statistical elements which limit the deterministic results naively hoped for a few years ago. However well an initial state of the atmosphere is known, C. Leith (National Center for Atmospheric Research, Boulder) reported, there is no basis for predicting atmospheric pressures beyond about two weeks. Likewise, the seismologist should not expect to interpret every detail on a seismogram, although $\mathbf{K}$. Aki (MIT) has used a standard backscattering theory to determine some interesting differences in the heterogeneity of California, Japan and Norway. Subsequent corridor discussion showed that scattering theories are being widely investigated, as, for different length scales of heterogeneity, completely different scattering modes may be appropriate. Even the standard formulations (based on acoustic waves) may give different asymptotic results for P-S scattering in the elastic case.

The effect of specified inhomogeneity on sufficiently long period waves should, of course, be deterministic, and quite realistic problems are now often attacked with the finite element method. A major computational advance here was reported by W. D. Smith (University of California, Berkeley), who has found a subtle way to eliminate unwanted reflections from the grid boundaries. He uses two sets of boundary conditions for which the sum of solutions sees the boundary as transparent, even for elastic (P-SV) waves. 